\title{
An ultrasensitive UPLC-MS/MS assay for the quantification of the therapeutic peptide liraglutide in plasma to assess the oral and nasal bioavailability in beagle dogs
}

\author{
Max Sauter', Philipp Uhl'1, Marius Majewsky', Margaux Fresnais², Walter E Haefeli \& \\ Jürgen Burhenne*,1 \\ ${ }^{1}$ Department of Clinical Pharmacology \& Pharmacoepidemiology, Heidelberg University Hospital, Im Neuenheimer Feld 410, 69120 \\ Heidelberg, Germany \\ ${ }^{2}$ Department of Clinical Pharmacology \& Pharmacoepidemiology, Heidelberg University Hospital, German Cancer Consortium \\ (DKTK), German Cancer Research Center (DKFZ), Im Neuenheimer Feld 410, 69120 Heidelberg, Germany \\ *Author for correspondence: Tel.: +49 622156 36395; Fax: +49 622156 5832; juergen.burhenne@med.uni-heidelberg.de
}

\begin{abstract}
Aim: An ultrasensitive UPLC-MS/MS assay for liraglutide was developed and validated according to US FDA and EMA guidelines and applied to the quantification of plasma concentrations after intravenous, nasal and oral administration of liraglutide to beagle dogs. Results: Liraglutide isolation was performed with a combined protein precipitation and solid-phase extraction protocol. The calibrated concentration range of $0.1-200 \mathrm{ng} / \mathrm{ml}$ was linear with correlation coefficients $>0.998$. Precise analysis was achieved through the utilization of an isotopically labeled internal standard. Absolute bioavailability of liraglutide after nasal and oral administration of liraglutide to beagle dogs was 0.03 and $0.006 \%$, respectively. Conclusion: The assay matches the performance in sensitivity of the previously applied immunoassay and optimally covers the therapeutic range of liraglutide.
\end{abstract}

First draft submitted: 7 January 2019; Accepted for publication: 4 April 2019; Published online: 16 May 2019

Keywords: bioavailability $\bullet$ liraglutide $\bullet$ nasal $\bullet$ oral $\bullet$ MS/MS $\bullet$ UPLC

Analogs of the endogenous GLP-1 are increasingly utilized and recommended early for the treatment of Type 2 diabetes mellitus [1]. GLP-1 is secreted mainly from L-cells of the gut following food intake and exhibits a half-life of approximately 2 min because of its rapid degradation by DPP-4 [2]. GLP-1 acts on GLP-1 receptors present in stomach, pancreas, brain and heart. It stimulates insulin secretion of pancreatic $\beta$-cells in the presence of hyperglycemia, delays gastric emptying, reduces appetite and exhibits positive effects on myocardial function [3]. Liraglutide, which is modified with a fatty acid for increased half-life and like all GLP-1 analogs shows elevated stability against DPP-4 degradation, is approved for the treatment of Type 2 diabetes mellitus and obesity [4].

Like most peptides, liraglutide has to be administered as subcutaneous injection because of its very low oral bioavailability. In order to avoid long-term injection therapy, alternative formulations enabling other routes of administration are desirable. To support development of novel noninjectable formulations of liraglutide, a highly sensitive and selective quantification assay is required. Traditionally, peptides are quantified using immunoassays because of the high sensitivity needed for reliable quantification of their usually low plasma concentrations. With the development of sensitive mass spectrometers, quantification of peptides is increasingly performed by MS/MS techniques because of their advantages of wider dynamic range, often great accuracy, and especially superior specificity because of the lack of crossreactivity [5].

Challenges arising in the MS/MS analysis of liraglutide are mainly because of its heavy molecular weight and numerous peptide bonds. The broad isotopic pattern and multiple charge distribution are lowering signal intensity of the MS signal while the resulting multitude of fragments in collision-induced decomposition (CID) is strongly diluting intensity in multiple reaction monitoring (MRM). 
A few MS/MS assays for the quantification of liraglutide in plasma have been reported relying on LC-MS/MS techniques [6-9]. The lowest LLOQ previously reported is $0.5 \mathrm{ng} / \mathrm{ml}$ when using $50 \mu \mathrm{l}$ of plasma for the quantification of liraglutide in rat plasma [9].

To support formulation development including the determination of the bioavailabilities after oral and nasal administration, we developed an ultrasensitive UPLC-MS/MS assay for the quantification of liraglutide in plasma. The assay with an LLOQ of $0.1 \mathrm{ng} / \mathrm{ml}$ using $100 \mu \mathrm{l}$ of plasma was fully validated according to US FDA and EMA pertinent guidelines $[10,11]$. It was subsequently applied to plasma samples of beagle dogs after oral, nasal and intravenous administration of liraglutide.

\section{Material \& methods}

Beagle study \& plasma sample generation

For the determination of the bioavailability of liraglutide after oral and nasal administration in beagle dogs, two studies were performed both conducted at commercial laboratories. The studies were carried out in accordance with national and European guidelines for the care and use of laboratory animals (European regulations; 2010/63/EU). The first study was performed at Charles River Laboratories Ltd (Tranent, Edinburgh, UK) under the UK Home Office Project License number PPL 70/8781. Four animals received an intravenous bolus injection of $0.5 \mathrm{mg}$ liraglutide in $0.5 \mathrm{ml}$ of phosphate-buffered saline (PBS). After a wash-out period of 2 weeks, the animals received $10 \mathrm{mg}$ of liraglutide filled into an enteric-coated capsule (Evonik Nutrition and Health, Darmstadt, Germany). Blood samples were taken into heparinized tubes $(0.8 \mathrm{ml})$ before administration and at $0.5,1,2,4,8$ and $24 \mathrm{~h}$ after intravenous injection as well as $0.5,1,2,3,4,8$ and $24 \mathrm{~h}$ after oral administration, immediately centrifuged at $3000 \times g$ for $10 \mathrm{~min}$ and the plasma was stored at $-80^{\circ} \mathrm{C}$ until analysis.

The second study was performed at LPT Laboratory of Pharmacology and Toxicology GmbH \& Co KG (Hamburg, Germany) under the German authorization number 33.2-42502-05-LG-01/2014/04 SA, approved by the Niedersächsisches Landesamt für Verbraucherschutz und Lebensmittelsicherheit, Oldenburg, Germany. Two animals received $0.8 \mathrm{mg}$ liraglutide as nasal administration comprising of two spray puffs (one spray puff of $0.1 \mathrm{ml}$ per nostril) of a $4 \mathrm{mg} / \mathrm{ml}$ liraglutide solution in PBS followed after a wash-out of 1 week by oral administration of $5 \mathrm{ml}$ of a $2 \mathrm{mg} / \mathrm{ml}$ solution of liraglutide in PBS. Blood samples were drawn into heparinized tubes $(0.8 \mathrm{ml})$ before and at $0.5,1,2,3,4,6,10$ and $24 \mathrm{~h}$ after administration, immediately centrifuged at $20,000 \times g$ for 2 min and the plasma was stored at $-20^{\circ} \mathrm{C}$ until analysis.

\section{Drugs, chemicals, solvents \& materials}

GMP-grade liraglutide was purchased from ChemWerth, Inc. (CT, USA). Isotopically labeled internal standard (IS) $\left({ }^{13} \mathrm{C}_{6},{ }^{15} \mathrm{~N}\right)$-Leu(26)-liraglutide was purchased from Peptide Specialty Laboratories GmbH (PSL; Heidelberg, Germany). Remaining reagents and solvents (water, methanol $[\mathrm{MeOH}]$, acetonitrile $[\mathrm{ACN}]$ and formic acid $[\mathrm{FA}]$ ) used for sample preparation, chromatography and MS were of the highest analytical quality (ULC/MS grade) and were purchased from Biosolve (Valkenswaard, The Netherlands). Blank beagle plasma for calibration, quality control (QC) and validation purposes was purchased from Innovative Research (MI, USA).

\section{Standard solutions}

For preparing calibration and QC stock solutions, liraglutide was independently weighed into low-bind reaction tubes and subsequently dissolved in $1 \mathrm{ml}$ of PBS because of increased solubility and to avoid adsorption loss observed for glass vessels. Solutions were diluted 100-fold with ACN/water $(1 / 1, v / v)+0.1 \%$ FA. Polypropylene tubes were used as vessels to prevent adsorption. A series of calibration subsolutions at concentrations of $0.1,0.4$, $1,3,10,30,100$ and $200 \mathrm{ng} / \mathrm{ml}$ were prepared by diluting the stock solution with $\mathrm{ACN} /$ water $(1 / 1, \mathrm{v} / \mathrm{v})+0.1 \%$ FA. QC subsolutions were prepared at the LLOQ $(0.1 \mathrm{ng} / \mathrm{ml})$ and at 0.275 (QC A), 5.5 (QC B), 110 (QC C) and $1000 \mathrm{ng} / \mathrm{ml}$ (dilution QC D).

The IS solution $\left(\left[{ }^{13} \mathrm{C}_{6},{ }^{15} \mathrm{~N}\right]\right.$-Leu(26)-liraglutide; $\left.90 \mathrm{ng} / \mathrm{ml}\right)$ was prepared accordingly. Solutions were kept at $4^{\circ} \mathrm{C}$. Stability of the solutions was proven for 3 month by quantification of 3-month-old QC solutions with freshly prepared calibration solutions.

\section{Plasma sample preparation}

Calibration and QC plasma samples $(100 \mu \mathrm{l})$ were spiked with $25 \mu \mathrm{l}$ of IS spike solution and $25 \mu \mathrm{l}$ of the respective calibration or QC spike solution in $2 \mathrm{ml}$ reaction tubes. Dilution QC samples were spiked with $25 \mu \mathrm{l}$ QC D solution 


\begin{tabular}{|c|c|c|}
\hline MS parameter & Liraglutide & {$\left[{ }^{13} \mathrm{C}_{6},{ }^{15} \mathrm{~N}\right]$-Leu(26)-liraglutide } \\
\hline Spray voltage & $2800 \mathrm{~V}$ & $2800 \mathrm{~V}$ \\
\hline Cone voltage & $50 \mathrm{~V}$ & $50 \mathrm{~V}$ \\
\hline Source temperature & $150^{\circ} \mathrm{C}$ & $150^{\circ} \mathrm{C}$ \\
\hline Desolvation gas flow $\left(\mathrm{N}_{2}\right)$ & $1000 \mathrm{I} / \mathrm{h}$ & $1000 \mathrm{I} / \mathrm{h}$ \\
\hline Desolvation temperature & $600^{\circ} \mathrm{C}$ & $600^{\circ} \mathrm{C}$ \\
\hline MRM transition $(m / z)$ & $938.8 \rightarrow 1064.1$ & $940.5 \rightarrow 1067.6$ \\
\hline Dwell time & $160 \mathrm{~ms}$ & $160 \mathrm{~ms}$ \\
\hline Collision energy & $22 \mathrm{~V}$ & $22 \mathrm{~V}$ \\
\hline Collision gas flow (Ar) & $0.15 \mathrm{ml} / \mathrm{min}$ & $0.15 \mathrm{ml} / \mathrm{min}$ \\
\hline
\end{tabular}

and subsequently $12.5 \mu \mathrm{l}$ added to $90 \mu \mathrm{l}$ blank plasma. Then $22.5 \mu \mathrm{ACN} /$ water $(1 / 19+0.1 \% \mathrm{FA}, \mathrm{v} / \mathrm{v})$ for volume substitution was added as well as $25 \mu \mathrm{l}$ IS. Study plasma samples $(100 \mu \mathrm{l})$ were also spiked with $25 \mu \mathrm{l}$ of IS and $25 \mu \mathrm{l}$ of ACN/water $(1 / 19+0.1 \% \mathrm{FA}, \mathrm{v} / \mathrm{v})$ for volume substitution. Subsequently, protein precipitation was performed on Impact ${ }^{\circledR}$ protein precipitation plates (Phenomenex, CA, USA) by addition of the plasma samples to the wells containing $300 \mu \mathrm{l}$ of $\mathrm{MeOH}$. The plate was sealed, shaken for $1 \mathrm{~min}$ and positive pressure applied (15 psi; positive pressure unit, Waters, MA, USA) to transfer protein-depleted extracts to a $2 \mathrm{ml} 96$-well collection plate (Eppendorf, Hamburg, Germany). To each well $350 \mu \mathrm{l}$ of $10 \%$ aqueous ammonia was added and gently mixed. $750 \mu \mathrm{l}$ of the samples were subsequently transferred to an anion exchange Oasis ${ }^{\circledR}$ MAX $\mu$-elution plate (Waters) that was operated with positive pressure. Wells were washed with $5 \%$ ammonia in $1 / 1 \mathrm{MeOH} /$ water followed by $20 \%$ aqueous ACN containing $0.05 \%$ FA. Elution of the samples into a 96 -well collection plate ( $800 \mu \mathrm{l}$; Waters) was performed with two times $30 \mu \mathrm{l}$ of $5 \% \mathrm{FA}$ in $1 / 1 \mathrm{ACN} /$ water. The collection plates were sealed and transferred into the Sample Manager for direct injection onto the UPLC-MS/MS system.

Instrumental analysis parameters

A Waters Xevo TQ-S with Z-spray ionization and step wave source optimization equipped with an Acquity I-class UPLC $^{\circledR}$ system consisting of a Waters Sample Manager flow-through-needle (FTN) and Binary Solvent Manager including cooled sample trays, integrated column heater and degasser was utilized for analysis.

A Waters Acquity BEH C18 column $(1.7 \mu \mathrm{m}, 2.1 \times 50 \mathrm{~mm})$ with integrated filter disc heated to $40^{\circ} \mathrm{C}$ was employed for UPLC separation. An injection volume of $20 \mu \mathrm{l}$ was utilized. Two eluents were used: eluent A consisting of $5 \%$ aqueous $\mathrm{ACN}$ with $0.1 \%$ (v) FA, and eluent $\mathrm{B}$ consisting of $\mathrm{ACN}$ containing $0.1 \%$ FA volume (v). The flow rate was $0.5 \mathrm{ml} / \mathrm{min}$ and was completely introduced in ion source. Chromatographic separation was achieved with a gradient starting at $80 \% \mathrm{~A} / 20 \% \mathrm{~B}$ for $0.1 \mathrm{~min}$, followed by a change of the eluent ratio to $5 \% \mathrm{~A} / 95 \% \mathrm{~B}$ within $2.4 \mathrm{~min}(0.1-2.5 \mathrm{~min})$. This ratio was then held for an additional $0.5 \mathrm{~min}$. To reduce carry-over the ratio of the eluents was subsequently changed to $95 \% \mathrm{~A} / 5 \% \mathrm{~B}$ within the next $0.5 \mathrm{~min}$, followed by a change to $5 \% \mathrm{~A} / 95 \% \mathrm{~B}$ within $0.5 \mathrm{~min}$. The system was finally reset to starting conditions within the following $0.5 \mathrm{~min}$. Equilibration of the UPLC system with the starting eluent ratio was performed for $1 \mathrm{~min}$ in which the Sample Manager set up the subsequent injection. This leads to a total cycle time of $5.5 \mathrm{~min}$.

Z-spray ionization parameters were manually optimized and the Xevo TQ-S was tuned automatically to liraglutide and the IS using MassLynx V4.1 system software (Waters) and the integrated IntelliStart standard procedures. MRM measurements were performed using argon as collision gas for CID with MS/MS transitions monitored in the positive ion mode. Mass spectrometric parameters are shown in Table 1.

\section{Validation of the analytical methods}

The assay was validated according to the guidelines of the FDA and EMA on bioanalytical method validation [10,11]. Accordingly, accuracy was determined in percent of the nominal value of the ratio of the mean concentrations measured in individual batches. Likewise, precision was calculated as percent of the determined standard deviation of the mean measured concentration. 
Validation was performed by the determination of three validation batches with each batch containing at least eight calibration samples and at least 24 QC samples at different concentrations (LLOQ, QC A, QC B and QC C, as well as additional dilution QC D; sixfold each). For proving the selectivity of the method blank plasma samples from six individual beagle dogs were measured and evaluated for interfering signals especially at the retention time of the analyte. Extraction recovery rates from plasma were determined from the peak areas of QC samples A, B and $\mathrm{C}$ compared with the respective peak areas obtained from blank plasma which was spiked with the corresponding amount of analyte after the extraction procedure. Matrix effects of plasma components potentially affecting the analysis were investigated by comparison of peak areas of blank plasma samples spiked after extraction at QC levels A, B and C with the respective peak areas of matrix-free UPLC solvent samples spiked with the identical amount [12].

Short-term and long-term stability of liraglutide in plasma at room temperature and $-80^{\circ} \mathrm{C}$ was not tested within this study because it has already been shown for the nanogram per milliliter range [7]. However, the stability of liraglutide in the nanogram per milliliter range was assessed for plasma samples at $-25^{\circ} \mathrm{C}$ as well as in three freeze-and-thaw cycles using QC samples A, B and C. Stability of the extracts in the autosampler was evaluated by repeated analysis of a 96-well plate after remaining in the autosampler at $10^{\circ} \mathrm{C}$ for $24 \mathrm{~h}$.

\section{Calculations \& statistical methods}

Calibration curves were determined by the ratio of the peak areas of the calibration samples analyte MRM transitions by the peak area of the IS MRM transition using weighted linear regression $\left(1 / \mathrm{x}^{2}\right)$ with Waters TargetLynx V4.1 software. Pharmacokinetic parameters were calculated with Thermo Kinetica Version 5.0 (Thermo Fisher Scientific, MA, USA) was applied for the calculation of pharmacokinetic parameters. Microsoft Office Excel 2010 (CA, USA) was used for the calculation of standard deviation, mean concentration and percent of nominal value.

\section{Results \& discussion}

\section{Choice of the LC-MS/MS system}

Because of the requirement of high sensitivity the development of the quantification assay was first performed on a microflow UPLC-MS/MS system (Acquity M-Class UPLC ${ }^{\circledR}$ system connected to a Xevo TQ-S equipped with an ionKey ${ }^{\circledR}$ inlet system; Waters). A BEH C18 (130 $\left.\AA\right)$ and BEH C4 (300 $\left.\AA\right)$ ionKey were tested for the chromatography of liraglutide. Although sharp peaks with a width at baseline of $4 \mathrm{~s}$ could be obtained, liraglutide showed such substantial carryover that specific analysis in reasonable time was impeded. Therefore, we decided to switch to UPLC and FTN injection to allow for rigorous washing of the LC-system, which was identified as the source of carry-over. The observed, extensive carry-over is thought to arise from the acylation with stearic acid present in liraglutide leading to elevated adsorption.

\section{Performance of the laboratory methods \\ Mass spectrometric \& chromatographic characteristics}

Positive electrospray ionization of liraglutide showed the maximum intensity for the $(\mathrm{M}+4 \mathrm{H})^{4+}$ signal at $m / z 938.8$, which was also observed for the IS at $\mathrm{m} / z$ 940.5. Figure 1 shows the respective daughter ion mass spectra and fragmentation reactions of the $(\mathrm{M}+4 \mathrm{H})^{4+}$ signal of liraglutide at $22 \mathrm{~V}$. The most intense CID fragments were observed at high collision energy $(80 \mathrm{~V})$ and at low $m / z$ values. These correspond to immonium ions of single amino acid residues with the most intense observed at $m / z 110.2$ which corresponds to the immonium ion of the $\mathrm{N}$-terminal histidine. Because high interference and background is more common for immonium ions due to their high abundance in interfering peptides also highly specific large fragments were investigated. The most intense larger fragment was observed at lower collision energies $(22 \mathrm{~V})$ at $m / z 1064.1$, which showed over a magnitude lower intensity than the most abundant immonium ions. Because the very high matrix interference for the immonium ion transitions such as the $\mathrm{m} / z 938.8 \rightarrow \mathrm{m} / z 110.2$ transition impeded sensitive analysis $(\mathrm{S} / \mathrm{N}$ ratio $<1$ at $0.2 \mathrm{ng} / \mathrm{ml})$, we chose the $\mathrm{m} / z 938.8 \rightarrow \mathrm{m} / z 1064.1$ transition for detection in the MRM which showed more than 40 -fold better $\mathrm{S} / \mathrm{N}$ values $(\mathrm{S} / \mathrm{N}$ ratio of 40 at $0.2 \mathrm{ng} / \mathrm{ml}$; Figure 2 ). The $\mathrm{m} / z 1064.1$ fragment constitutes the doubly charged C-terminal fragment after cleavage of the peptide bond between positions 15 and 16 ( $\mathrm{y}_{16}$-fragment, Figure 1 ) which was confirmed with high-resolution mass spectrometry using a Waters Xevo G2-XS QTof: The found monoisotopic $m / z 1063.6282$ deviated less than 0.2 p.p.m. from the calculated monoisotopic $m / z 1063.6284$ of the $\mathrm{y}_{16}$-fragment. For the isotopically labeled IS, the corresponding mass transition at $m / z 940.5 \rightarrow m / z 1067.6$ was monitored. The mass shift of $3.5 \mathrm{Da}$ of the $m / z 1067.6$ fragment of the IS in 


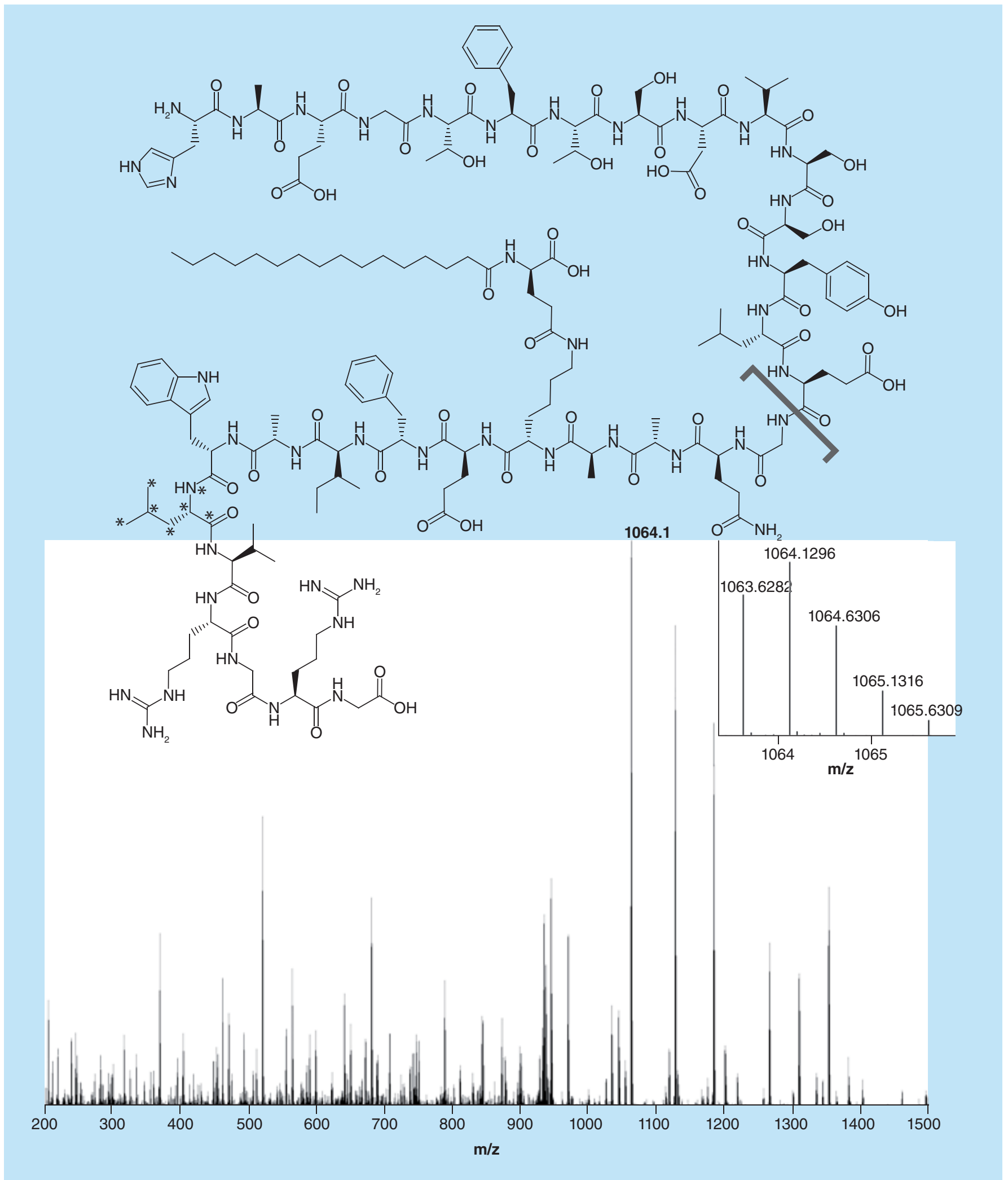

Figure 1. Positive product spectrum of liraglutide using collision-induced decomposition at $22 \mathrm{~V}$. The grey bar in the liraglutide structure depicts the $m / z 938.8 \rightarrow m / z$ 1064.1. The positions of the isotopic labels in the corresponding internal standard are tagged with stars. 
(A)

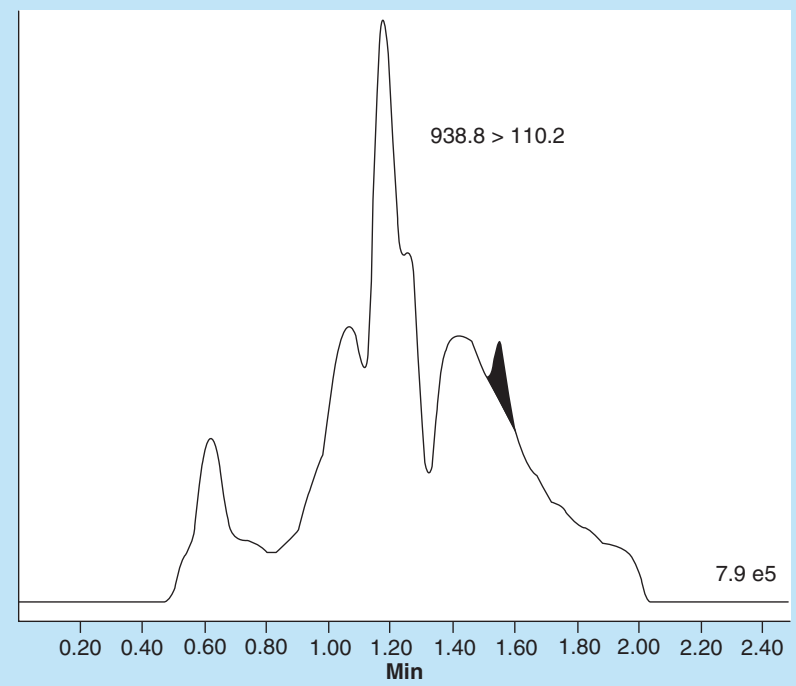

(B)

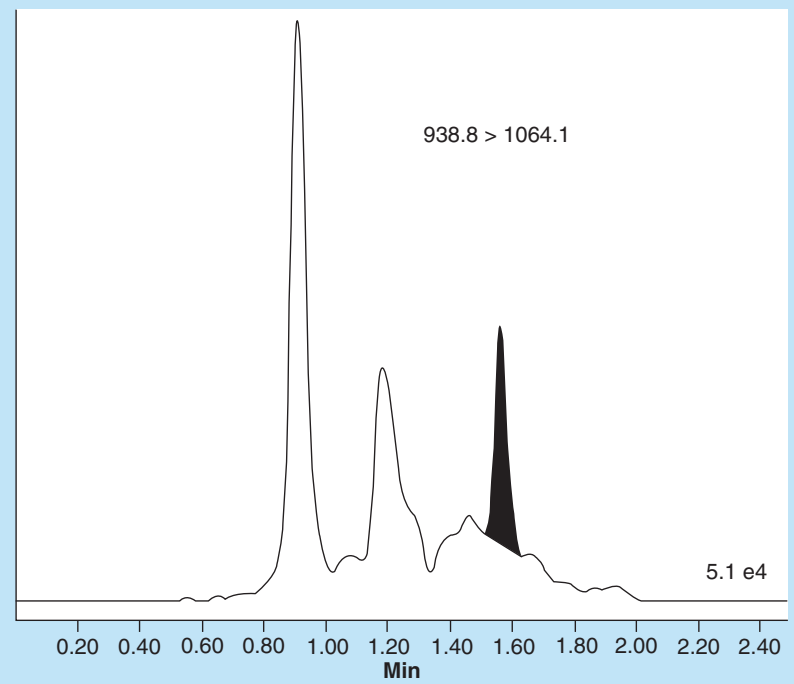

Figure 2. Quantification of a plasma sample containing $0.2 \mathrm{ng} / \mathrm{ml}$ liraglutide. (A) Monitoring of the $\mathrm{m} / \mathrm{z} 938.8 \rightarrow \mathrm{m} / \mathrm{z} 110.2 \mathrm{transition}$ and (B) monitoring of the $m / z 938.8 \rightarrow m / z 1064.1$ transition.

comparison to the $m / z 1064.1$ fragment of the analyte is in line with the doubly charged $\mathrm{y}_{16}$-fragment comprising the isotopically labeled $\left({ }^{13} \mathrm{C}_{6},{ }^{15} \mathrm{~N}\right.$ )-leucine (Figure 1$)$.

UPLC was performed on a Waters BEH C18 UPLC ${ }^{\circledR}$ column with a gradient from 80 to $5 \%$ aqueous eluent and a parallel increase of the ACN eluent from 20 to $95 \%$ resulting in a fast separation of only 2 min and a peak width at baseline of $6 \mathrm{~s}$. Well resolved single mass traces were achieved with dwell times of $160 \mathrm{~ms}$ for each transition yielding 20 analysis points per peak (Figure 3). Initially, considerable carryover was observed for liraglutide, which could be reduced to levels not affecting the analysis by keeping the ACN eluent at $95 \%$ for 0.5 min after the separation gradient and the subsequent run of an additional cleaning gradient. For this cleaning gradient the ACN fraction was reduced to $5 \%$ in $0.5 \mathrm{~min}$ followed by an increase to $95 \%$ in $0.5 \mathrm{~min}$ and subsequently returned to starting conditions of $20 \% \mathrm{ACN}$ within $0.5 \mathrm{~min}$. Equilibration of starting conditions was performed for $1 \mathrm{~min}$ while the Sample Manager prepared the following injection which resulted in a total cycle time of $5.5 \mathrm{~min}$.

\section{Extraction by protein precipitation}

Isolation of liraglutide was achieved by protein precipitation with a three-time excess of $\mathrm{MeOH}$ over plasma, followed by solid phase extraction (SPE) using mixed mode strong anion exchange and reverse phase sorbent. $\mathrm{MeOH}$ was used for protein depletion because it showed substantially higher recoveries when compared with ACN. To enable enrichment of liraglutide in the extracts, anion exchange purification was performed on a $\mu$-elution plate allowing elution with volumes as little as two times $30 \mu \mathrm{l}$. Endogenous components interfered substantially in analyses of protein precipitation extracts of liraglutide while anion exchange purification efficiently removed matrix interference likely because of the optimized rigorous washing steps. $5 \%$ ammonia in $1 / 1 \mathrm{MeOH} /$ water removed basic compounds as well as substances with significant retention on the reverse phase. The following washing step with slightly acidic $20 \%$ aqueous ACN aimed at removing slightly acidic substances while elution of liraglutide was avoided. Because of these optimized washing steps, matrix interference in the analysis of liraglutide was significantly reduced.

Plasma recovery rates for the isolation procedure were assessed in triplicate determination at QC levels A, B and C as well as for IS. Recoveries of IS and QC samples after solely performed protein precipitation ranged between 53.9 and $64.5 \%$ while additional anion exchange extraction reduced recoveries to $36.3-39.0 \%$. Remaining components of biological matrix such as peptides and lipids may influence baseline stability of the chromatography and/or induce unexpected peaks, and may alter the ionization process by ion suppression/enhancement, which can result in decreased accuracy and precision particularly at low concentrations [12]. The more efficient separation of matrix 


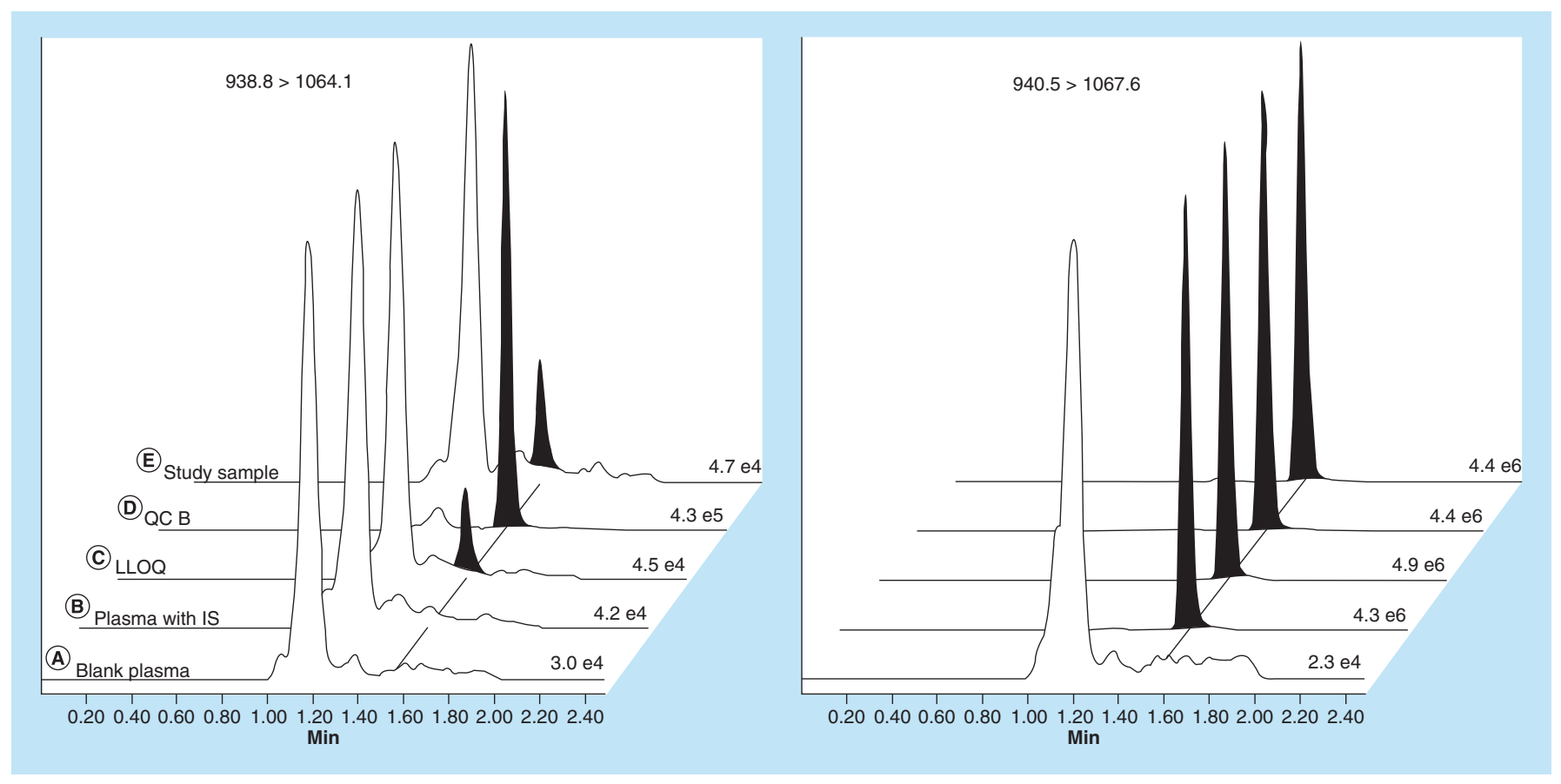

Figure 3. Selected UPLC-MS/MS chromatograms of processed plasma samples with liraglutide transition (left) and internal standard transition (right). (A) Blank plasma sample, (B) plasma sample with added IS, (C) plasma sample at LLOQ concentration (representing $0.1 \mathrm{ng} / \mathrm{ml}$, (D) plasma sample at QC B concentration (representing $5.5 \mathrm{ng} / \mathrm{ml}$ ) and (E) plasma sample of beagle dog number $5,6 \mathrm{~h}$ after nasal administration of $0.8 \mathrm{mg}$ liraglutide (quantified liraglutide concentration $0.13 \mathrm{ng} / \mathrm{ml}$ ).

IS: Internal standard; QC: Quality control.

with optimized anion exchange extraction improved the matrix effect to ranges between -20.4 and $-31.2 \%$ as compared with protein-depleted extracts, which showed matrix effects of -30.0 to $-41.7 \%$. Taking into account the enrichment of liraglutide in the extracts by the $\mu$-elution SPE procedure, the amount of liraglutide injected onto the UPLC-MS/MS system is larger after SPE. Because of the enrichment by the SPE procedure the assay holds the perspective for lowering the LLOQ when using larger amounts of plasma. Additionally, the $\mathrm{S} / \mathrm{N}$ ratio was maximized by the anion exchange extraction procedure because of significantly reduced matrix interference. Analysis of QC B samples of lipemic and hemolytic plasma revealed no influence on sample analysis with respective accuracies of 93.0 and $89.5 \%$. Although lipemic QC plasma samples showed peak areas similar to normal plasma, hemolytic plasma yielded substantially lower peak areas. This may either be attributed to decreased recovery or an increased matrix effect. Therefore, analysis of hemolytic plasma samples at low concentrations should be avoided.

\section{Validation results}

The isolation by protein precipitation followed by anion exchange extraction combined with UPLC-MS/MS quantification entirely met FDA's and EMA's requirements for bioanalytical method validation $[10,11]$. The absence of interfering signals in blank matrices from six different beagle dogs confirmed the selectivity of the assay. Figure 3 shows typical chromatograms of blank plasma as well as plasma samples spiked with IS, at LLOQ, at QC B, and beagle plasma $6 \mathrm{~h}$ after nasal administration of $0.8 \mathrm{mg}$ liraglutide. Correlation coefficients $\left(\mathrm{r}^{2}\right)$ of $\geq 0.998$ (linear regression and $1 / \mathrm{x}^{2}$ weighting) were achieved within the calibrated range $(0.1-200 \mathrm{ng} / \mathrm{ml})$. The dynamic range of the assay optimally covers the therapeutic range of liraglutide in humans and matches the performance of the previously described immunoassay for pharmacokinetic analysis $(0.068-16.9 \mathrm{ng} / \mathrm{ml})$ [13]. Within-batch accuracies (QC A-C) varied between 93.8 and $105.4 \%$ and the batch-to-batch accuracies ranged from 96.7 to $101.4 \%$ showing precision values of $<6.2 \%$. Accuracy at LLOQ level varied between 92.8 and $106.3 \%$ within-batch and was $98.7 \%$ batch-to-batch with corresponding precision values of $<10.7 \%$. Accuracy and precision values are shown in Table 2 . This precise intra- and interday quantification is attributed to the quantification using isotopically labeled IS that completely accounts for matrix effects and recovery. 
Table 2. Summary of quality control results for liraglutide in plasma.

\begin{tabular}{|c|c|c|c|c|c|}
\hline Quality control criteria & & LLOQ $(0.100 \mathrm{ng} / \mathrm{ml})$ & QC A $(0.275 \mathrm{ng} / \mathrm{ml})$ & QC B $(5.50 \mathrm{ng} / \mathrm{ml})$ & QC C (110 ng/ml) \\
\hline \multicolumn{6}{|l|}{ Within batch } \\
\hline \multirow[t]{3}{*}{1} & Mean (ng/ml) & 0.093 & 0.267 & 5.39 & 112 \\
\hline & Accuracy (\%) & 92.8 & 96.9 & 98.0 & 101.8 \\
\hline & Precision (\%CV) & 7.57 & 3.26 & 3.52 & 4.26 \\
\hline \multirow[t]{3}{*}{2} & Mean (ng/ml) & 0.097 & 0.290 & 5.40 & 112 \\
\hline & Accuracy (\%) & 96.8 & 105.4 & 98.1 & 101.7 \\
\hline & Precision (\%CV) & 10.7 & 3.69 & 2.64 & 2.14 \\
\hline \multirow[t]{3}{*}{3} & Mean (ng/ml) & 0.106 & 0.260 & 5.16 & 111 \\
\hline & Accuracy (\%) & 106.3 & 94.5 & 93.8 & 100.8 \\
\hline & Precision (\%CV) & 8.38 & 5.18 & 1.18 & 1.69 \\
\hline \multicolumn{6}{|l|}{ Batch-to-batch } \\
\hline & Mean (ng/ml) & 0.099 & 0.272 & 5.32 & 112 \\
\hline & Accuracy (\%) & 98.7 & 98.9 & 96.7 & 101.4 \\
\hline & Precision (\%CV) & 10.3 & 6.20 & 3.28 & 2.78 \\
\hline
\end{tabular}

For quantification of intravenously administered liraglutide, a tenfold dilution with blank plasma (QC D) was validated using $10 \mu \mathrm{l}$ of plasma. Accuracy of the dilution QC D was $106.9 \%$, hence the assay can be applied to quantify liraglutide in plasma in a range of $0.1-2000 \mathrm{ng} / \mathrm{ml}$. Additionally, the incurred sample reanalysis of 25 beagle samples met FDA's and EMA's requirements with $84 \%$ of the samples showing a deviation of $<20 \%$.

\section{Stability}

Stability in the nanogram per milliliter range was tested within our validation procedure using three freeze-and-thaw cycles and samples stored at $-25^{\circ} \mathrm{C}$ for 2 month for QC A, B and C plasma samples. Accuracies of the freeze-andthaw samples ranged between 103.3 and $110.4 \%$, confirming the stability of liraglutide during the freeze-and-thaw cycles. Further, QC A, B and C samples stored at $-25^{\circ} \mathrm{C}$ for 2 months showed accuracies ranging from 108.9 to $110.4 \%$. Liraglutide is, therefore, considered stable in plasma for at least 2 months at $-25^{\circ} \mathrm{C}$. Stability in plasma has previously been demonstrated for 1 week at $-80^{\circ} \mathrm{C}$ and $4 \mathrm{~h}$ at room temperature for the nanogram per milliliter range [7]. Additionally, plasma extracts were stable after remaining in the Sampler Manager for $24 \mathrm{~h}$. The stability of liraglutide in the stock and standard solutions was confirmed by the quantification of a freshly prepared stock solution after 3 month. The prepared QC A, B and C samples of this fresh stock solution showed accuracies ranging from 105.1 to $113.1 \%$.

Pharmacokinetics of liraglutide after nasal, oral \& intravenous administration to beagle dogs Because of the acylation with palmitic acid, liraglutide exhibits a relatively long half-life of $8 \mathrm{~h}$ in humans after intravenous injection [14]. The half-life determined in beagle dogs after intravenous injection was quite similar with $9.4 \mathrm{~h}$ and comparable after oral administration with $8.4 \mathrm{~h}$. The volume of distribution after intravenous and oral administration of $0.057 \mathrm{l} / \mathrm{kg}$ and $0.070 \mathrm{l} / \mathrm{kg}$, respectively, was also comparable to the reported values in humans of $0.07 \mathrm{l} / \mathrm{kg}$ [14]. This similarity in the pharmacokinetic characteristics is most likely because of the extensive protein binding of liraglutide of $>98 \%$ [15] in both species. Pharmacokinetic characteristics in beagle dogs after intravenous, nasal and oral administration are shown in Table 3.

The oral bioavailability of liraglutide when administered in solution was determined as $0.006 \%$. After oral administration of $10 \mathrm{mg}$ liraglutide in enteric-coated capsules for protection from proteolysis in the stomach, no detectable plasma concentrations were observed. This indicates an absolute bioavailability of less than $0.001 \%$ which was estimated to represent the minimum detectable $C_{\max }$ value when assuming a similar pharmacokinetic profile as observed for oral liraglutide in solution. This may be attributed to a delayed release of liraglutide from the enteric-coated capsule or a solubility issue of the solid liraglutide.

Nasal administration of $0.8 \mathrm{mg}$ liraglutide resulted in detectable plasma concentrations of up to $0.13 \mathrm{ng} / \mathrm{ml}$. Nasal absorption appeared very slow with $\mathrm{C}_{\max }$ values reached 6-10 h after administration (Figure 4). Although 
Table 3. Single dose pharmacokinetics of liraglutide in beagle dogs after intravenous bolus injection and after oral administration in solution.

\begin{tabular}{|c|c|c|c|c|c|c|c|}
\hline $\begin{array}{l}\text { Liraglutide dose and route } \\
\text { of administration }\end{array}$ & Dog number & $T_{1 / 2}(h)$ & $C_{\max }(\mathrm{ng} / \mathrm{ml})$ & $\begin{array}{l}\operatorname{AUC}_{\mathrm{Inf}}(\mathrm{h} \times \\
\mathrm{ng} / \mathrm{ml})\end{array}$ & $F(\%)$ & $V_{z}(I / k g)$ & $\mathrm{Cl}(\mathrm{ml} / \mathrm{min})$ \\
\hline \multirow[t]{3}{*}{ Intravenous $0.5 \mathrm{mg}$} & 1 & 9.15 & 984 & 10330 & 100 & 0.048 & 0.81 \\
\hline & 3 & 10.4 & 944 & 11785 & 100 & 0.060 & 0.71 \\
\hline & 4 & 8.83 & 823 & 8594 & 100 & 0.060 & 0.97 \\
\hline \multirow[t]{2}{*}{ Nasal $0.8 \mathrm{mg}$} & 5 & 35.0 & 0.13 & 6.13 & 0.039 & 0.17 & 0.66 \\
\hline & 6 & 19.3 & 0.12 & 3.42 & 0.022 & 0.17 & 1.19 \\
\hline \multirow[t]{2}{*}{ Oral $10 \mathrm{mg}$} & 7 & 8.90 & 1.26 & 19.2 & 0.010 & 0.071 & 0.65 \\
\hline & 8 & 10.5 & 0.25 & 4.07 & 0.002 & 0.070 & 0.77 \\
\hline
\end{tabular}

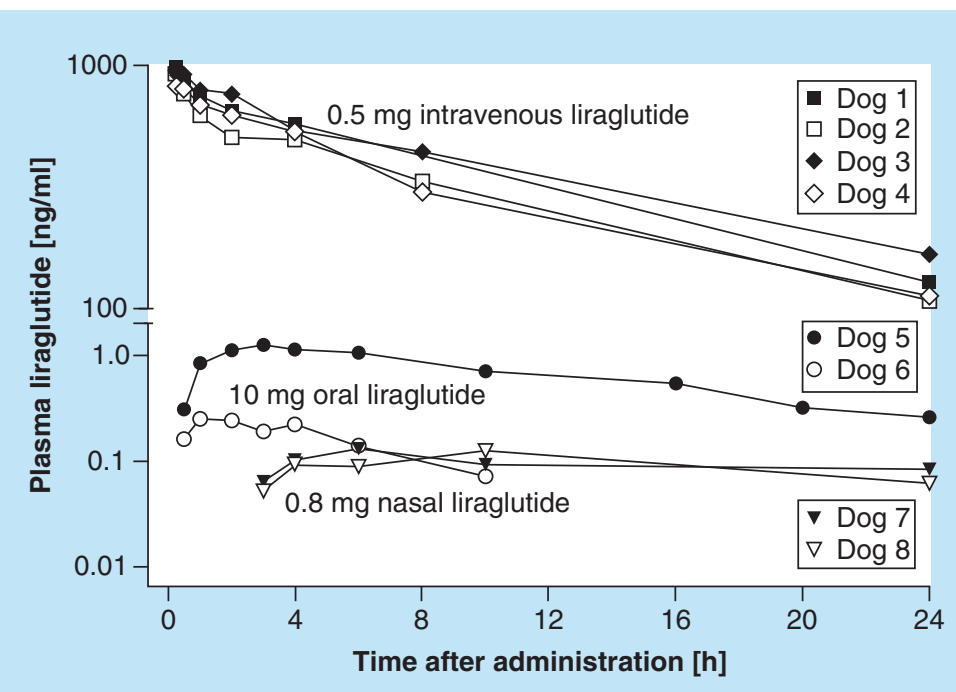

Figure 4. Plasma concentration-time profiles of liraglutide after intravenous, nasal and oral administration in solution. The upper curves represent the single intravenous bolus injection of $0.5 \mathrm{mg}$ liraglutide to beagle dogs number 1-4. The middle curves correspond to oral administration of $10 \mathrm{mg}$ liraglutide to beagle dogs numbers 5 and 6. The lower curves represent the nasal administration of $0.8 \mathrm{mg}$ liraglutide to beagle dogs numbers 7 and 8 .

clearance was comparable regardless of the route of administration, the half-life after nasal administration of liraglutide was prolonged in comparison to oral and intravenous administration with approximately $27 \mathrm{~h}$ and the volume of distribution was also substantially larger with $0.17 \mathrm{l} / \mathrm{kg}$ which both is attributed to the slow absorption from the nasal cavity. The nasal bioavailability was calculated as $0.03 \%$. Minimal oral and nasal bioavailability is not uncommon for peptides because of their instability against proteolytic digestion and poor membrane penetration [16].

\section{Conclusion}

Only few assays for the quantification of liraglutide in plasma using LC-MS/MS have been described with the most sensitive having an LLOQ of $0.5 \mathrm{ng} / \mathrm{ml}$ when using $50 \mu \mathrm{l}$ of plasma. None of these assays used an isotopically labeled IS. In this paper, we describe a novel ultrasensitive UPLC-MS/MS assay with an LLOQ of $0.1 \mathrm{ng} / \mathrm{ml}$ (27 picomol/l) when using a plasma volume of $100 \mu \mathrm{l}$. This is the most sensitive assay to date and also the first utilizing an isotopically labeled IS which resulted in excellent accuracy ( $<3.3 \%$ batch-to-batch). The improvement in sensitivity could be achieved by enrichment of liraglutide in plasma extracts while concurrently minimizing matrix interference because of an optimized SPE procedure. The utilization of $\mu$-elution SPE in 96-well format for sample 
extraction allowed rigorous washing which efficiently minimized matrix interference and enabled enrichment of liraglutide in the extracts by elution in small volumes. This sample enrichment also holds the possibility of lowering the LLOQ when using larger amounts of plasma. Further, combination of UPLC with a sensitive MS/MS enabled high sensitivity in MRM while optimized chromatographic conditions facilitated short chromatograms (2 min) with sharp peaks to further maximize intensity and $\mathrm{S} / \mathrm{N}$ ratio as well as separating interfering signals. The assay was successfully applied to plasma samples of beagle dogs that received liraglutide by nasal, oral and intravenous administration allowing the determination of the corresponding bioavailabilities.

\section{Future perspective}

Bioanalysis of peptides is increasingly performed by LC-MS/MS replacing the traditionally performed highly sensitive immunoassays. LC-MS/MS is usually faster in the development and shows superior dynamic range and selectivity. The here described assay using readily available equipment, matching the performance in sensitivity of the previously applied immunoassay, is a step toward routine bioanalysis of peptides with high potency and, therefore, low plasma concentrations by ultrasensitive LC-MS/MS methodologies.

\section{Summary points}

\section{Aim}

- GLP-1 analogs including the therapeutic peptide liraglutide are increasingly utilized and recommended early in the treatment of Type 2 diabetes mellitus. Like most peptides liraglutide needs to be administered by injection.

- To support future development of alternative routes of administration an ultrasensitive UPLC-MS/MS assay for the quantification of liraglutide in plasma is required. The ultrasensitive bioanalysis of liraglutide by MS/MS is challenging because of its high molecular weight which leads to signal dilution in collision induced decomposition.

Results \& discussion

- An ultrasensitive UPLC-MS/MS assay using multiple reaction monitoring and positive electrospray ionization was developed entirely complying with US FDA's and EMA's guidelines on bioanalytical method validation.

- Because of substantial adsorption characteristics of liraglutide its bioanalysis on a microflow UPLC was impeded and was therefore performed on a UPLC with flow-through-needle injection allowing for rigorous washing which reduced carryover to levels not affecting the assay's specificity.

- Extraction of liraglutide from plasma was achieved by combining protein precipitation and solid-phase extraction. This efficiently removed endogenous interference because of rigorous washing steps while it concentrated the samples through $\mu$-elution.

- This assay has the lowest reported LLOQ of $0.1 \mathrm{ng} / \mathrm{ml}$ (when using $100 \mu \mathrm{l}$ of plasma) matching the performance of the previously described immunoassay and optimally covers the therapeutic range of liraglutide. It is the first assay for the quantification of liraglutide utilizing an isotopically labeled internal standard which resulted in precise analysis showing excellent interday accuracy.

- This assay was applied for the determination of liraglutide pharmacokinetics and bioavailability after intravenous, nasal and oral administration to beagle dogs. Absolute bioavailability of liraglutide after oral and nasal administration was 0.006 and $0.03 \%$, respectively.

Conclusion

- To our knowledge, this is the most sensitive assay for the quantification of liraglutide using MS/MS also showing excellent accuracy and precision and was successfully applied to a pharmacokinetic study in beagle dogs.

\section{Acknowledgements}

The authors would like to thank M Arndt (Evonik Nutrition and Health, Darmstadt, Germany) for helpful suggestions on the conduct of the beagle dog studies.

\section{Author's contributions}

WE Haefeli, J Burhenne, M Sauter and P Uhl conceived the study, planned the experiments and performed the data analysis. P Uhl prepared the samples (capsules, solutions and spray) for administration in the animal studies. M Sauter developed and validated the UPLC-MS/MS assay, measured the animal study plasma samples and wrote the manuscript. M Majewsky supported chromatography development. M Fresnais supported HRMS analysis for fragment identification. 
Financial \& competing interests disclosure

The authors appreciate the funding by the Bundesministerium für Bildung und Forschung (grant number 03VP03980). The authors declare no conflict of interest. The authors have no other relevant affiliations or financial involvement with any organization or entity with a financial interest in or financial conflict with the subject matter or materials discussed in the manuscript apart from those disclosed.

No writing assistance was utilized in the production of this manuscript.

Ethical conduct of research

The authors state that they have obtained appropriate institutional review board approval or have followed the principles outlined in the Declaration of Helsinki for all human or animal experimental investigations. Animal studies were carried out in accordance with national and European guidelines for the care and use of laboratory animals (European regulations; 2010/63/EU) and approved by the pertinent national authorities.

\section{References}

Papers of special note have been highlighted as: • of interest; $\bullet \bullet$ of considerable interest

1. Garber AJ, Abrahamson MJ, Barzilay JI et al. Consensus statement by the American Association of Clinical Endocrinologists and American College of Endocrinology on the comprehensive Type 2 diabetes management algorithm - 2018 executive summary. Endocr. Pract. 24(1), 91-120 (2018).

2. Vilsboll T, Agersø H, Krarup T, Holst JJ. Similar elimination rates of glucagon-like peptide-1 in obese Type 2 diabetic patients and healthy subjects. J. Clin. Endocrinol. Metab. 88(1), 220-224 (2003).

3. Russell S. Incretin-based therapies for Type 2 diabetes mellitus: a review of direct comparisons of efficacy, safety and patient satisfaction. Int. J. Clin. Pharm. 35(2), 159-172 (2013).

4. Mancini MC, De Melo ME. The burden of obesity in the current world and the new treatments available: focus on liraglutide $3.0 \mathrm{mg}$. Diabetol. Metab. Syndr. 9, 44 (2017).

5. Lovgren U, Johansson S, Jensen LS, Ekstrom C, Carlshaf A. Quantitative determination of peptide drug in human plasma samples at low $\mathrm{pg} / \mathrm{ml}$ levels using coupled column liquid chromatography-tandem mass spectrometry. J. Pharm. Biomed. Anal. 53(3), 537-545 (2010).

6. Kellie JF, Kehler JR, Szapacs ME. Application of high-resolution MS for development of peptide and large-molecule drug candidates. Bioanalysis 8(3), 169-177 (2016).

- First report of the bioanalysis of liraglutide using LC-MS.

7. Meng X, Xu H, Zhang $\mathrm{Z}$ et al. Differential mobility spectrometry tandem mass spectrometry with multiple ion monitoring for the bioanalysis of liraglutide. Anal. Bioanal. Chem. 409(20), 4885-4891 (2017).

- First report of the bioanalysis of liraglutide using LC-MS/MS. Parent to parent pseudo-multiple reaction monitoring was performed after ion mobility.

8. King-Ahmad AJ, Kalgutkar AS, Niosi M, Eng H, Holliman C. A sensitive method for the quantitation of the peptide-based glucagon-like peptide-1 receptor agonist liraglutide in plasma using microfluidics chromatography tandem MS. Bioanalysis 10(5), 357-368 (2018).

- First report of the bioanalysis of liraglutide using LC-MS/MS with multiple reaction monitoring.

9. Dong S, Gu Y, Wei G, Si D, Liu C. Determination of liraglutide in rat plasma by a selective liquid chromatography-tandem mass spectrometry method: application to a pharmacokinetics study. J. Chromatogr. B. Analyt. Technol. Biomed. Life Sci. 1091, 29-35 (2018).

- Most sensitive assay for the bioanalysis of liraglutide using LC-MS/MS previously developed.

10. US Department of Health and Human Services, Food and Drug Administration (FDA), Center for Drug Evaluation and Research (CDER). Guidance for industry, bioanalytical method validation (2018). www.fda.gov/downloads/Drugs/GuidanceComplianceRegulatoryInformation/Guidances/ucm070107.pdf

11. The European Medicines Agency (EMA), Committee for Medicinal Products for Human Use. Guideline on bioanalytical method validation (2011). www.ema.europa.eu/docs/enGB/documentlibrary/Scientificguideline/2011/08/WC500109686.pdf

12. Matuszewski BK, Constanzer ML, Chavez-Eng CM. Strategies for the assessment of matrix effect in quantitative bioanalytical methods based on HPLC-MS/MS. Anal. Chem. 75, 3019-3030 (2003).

13. Agersø H, Jensen LB, Elbrønd B, Rolan P, Zdravkovic M. The pharmacokinetics, pharmacodynamics, safety and tolerability of NN2211, a new long-acting GLP-1 derivative, in healthy men. Diabetologia 45(2), 195-202 (2002).

-• Description of immunoassay for the bioanalysis of liraglutide and pharmacokinetics after repeated dose.

14. Elbrønd B, Jakobsen G, Larsen S et al. Pharmacokinetics, pharmacodynamics, safety, and tolerability of a single-dose of NN2211, a long-acting glucagon-like peptide 1 derivative, in healthy male subjects. Diabetes Care 25(8), 1398-1404 (2002).

- Single-dose pharmacokinetics of liraglutide in human subjects.

15. Plum A, Jensen LB, Kristensen JB. In vitro protein binding of liraglutide in human plasma determined by reiterated stepwise equilibrium dialysis. J. Pharm. Sci. 102(8), 2882-2888 (2013). 
- Evaluation of the adsorption characteristics of liraglutide and circumvention of those for the determination of its protein binding.

16. Rembratt A, Graugaard-Jensen C, Senderovitz T, Norgaard JP, Djurhuus JC. Pharmacokinetics and pharmacodynamics of desmopressin administered orally versus intravenously at daytime versus night-time in healthy men aged 55-70 years. Eur. J. Clin. Pharmacol. 60(6), 397-402 (2004). 